\title{
A straightforward estimation of cardiac substructure exposure for clinical practice: example of breast rotational intensity modulated radiation therapy
}

\author{
Pierre Loap, Youlia Kirova
}

Institut Curie, Paris, France

\begin{abstract}
Introduction. Mean heart dose (MHD) is the most widely used dosimetric parameter for cardiac sparing during treatment planning. Specific cardiac substructure exposure could be more clinically important, but MHD cannot provide the radiation oncologist with precise insight at the substructural level.

Materials. We propose a straightforward method for estimating cardiac substructure exposure based on linear regressions between mean dose delivered to cardiac substructures and MHD. We focused on breast irradiation with intensity modulated radiation therapy as an application example. Correlations between mean dose to cardiac substructures and MHD were statistically significant and usually moderate $(r>0.5)$ or strong $(r>0.7)$, allowing the use of such linear regression models to estimate cardiac substructure exposure from MHD for clinical practice.
\end{abstract}

Conclusion. This method can be extrapolated to other clinical situations for daily practice, albeit with some restrictions.

NOWOTWORY J Oncol 2020; 70, 3: 98-100

Key words: cardiac substructure exposure, cardiotoxicity, intensity modulated radiation therapy, breast cancer

\section{Introduction}

Adjuvant radiation therapy decreases breast cancer mortality but poses cardiotoxicity risks [1]. Fortunately, recent techniques efficiently spare the heart [2], such as rotational intensity modulated radiation therapy (IMRT) or proton radiation therapy. However, the heart is a complex organ made of multiple histologically-diverse substructures and the widely used mean heart dose (MHD) cannot provide a precise insight at the substructural level. In this perspective, we propose a straightforward method for estimating mean doses to cardiac substructures when only MHD is known (which is the case for clinical practice), focusing on locoregional breast cancer irradiation using rotational IMRT as an application example.

\section{Materials}

Thirty breast cancer patients having undergone breast conservation surgery and subsequently irradiated with rotational IMRT (volumetric modulated arc therapy or helical tomotherapy) were selected by random sampling from our institutional database. Sixteen patients were treated for left-sided breast cancers and 14 for right-sided breast cancers. Target volumes included the whole breast with a boost, homolateral axillary lymph nodes and internal mammary chain. Half of the radiation schedules were hypofractionated: hypofractionated regimens delivered 52.2 to 56 Gy to the tumour bed (in 18 to 23 fractions) while normofractionated regimens delivered 63 to 66 Gy. Cardiac substructures were delineated according to published international guidelines [3]. Mean doses to the heart (MHD),

\section{How to cite:}

Loap P, Kirova Y. A straightforward estimation of cardiac substructure exposure for clinical practice: example of breast rotational intensity modulated radiation therapy. NOWOTWORY J Oncol 2020; 70: 98-100. 
cardiac cavities, left ventricular walls and coronary arteries were retrieved. For each substructure, a simple linear regression was performed between the mean dose to the considered cardiac substructure and the MHD.

\section{Results}

For all substructures, MHD was a significant explanatory variable (with the exception of the first two right coronary artery segments for left-sided irradiation). The cardiac substructures which were the most sensitive to MHD variation were the left ventricle anterior wall $(\beta=1.8, p<0.001)$ for left-sided irradiations and the right auricle $(\beta=1.3, p<0.001)$ for right-sided irradiations. The coronary artery segments which were the most sensitive to MHD variation were the mid segment of the left anterior descending coronary artery $(\beta=2.9, p<0.001)$ for left-sided irradiations and the proximal segment of the right coronary artery $(\beta=1.6, p<0.001)$ for right-sided irradiations.

For right-sided irradiations, the correlations were usually strong $(r>0.7)$. Correlations were somewhat weaker for left-sided irradiations, but the correlation coefficient was still usually greater than 0.5. The greatest correlation with the MHD was found for the distal circumflex artery segment for left-sided irradiations $(r=0.88, p<0.001)$ and for the left ventricle septal wall $(r=0.95, p<0.001)$ for right-sided irradiations.

\section{Discussion}

Based on simple linear regressions, this method for estimating cardiac substructure exposure is straightforward and convenient when only MHD is available, which is often the case in daily practice since cardiac substructures are usually not

Table I. Provides the equations estimating the mean dose delivered to each substructure from MHD

a. Linear models for cardiac substructure

\begin{tabular}{|c|c|c|c|c|c|c|}
\hline & \multicolumn{6}{|c|}{ Cardiac substructure } \\
\hline & \multicolumn{3}{|c|}{ Left-sided breast irradiation } & \multicolumn{3}{|c|}{ Right-sided breast irradiation } \\
\hline & Mean dose & $r$ & p value & Mean dose & $\mathbf{r}$ & p value \\
\hline Left ventricle (LV) & $=1.0 \times \mathrm{MHD}-0.4$ & 0.84 & $<0.001$ & $=0.7 \times M H D-0.7$ & 0.95 & $<0.001$ \\
\hline LV anterior wall & $=1.8 \times \mathrm{MHD}-2.1$ & 0.73 & $<0.001$ & $=0.7 \times M H D+0.1$ & 0.81 & $<0.001$ \\
\hline LV apical wall & $=1.4 \times \mathrm{MHD}+0.7$ & 0.35 & 0.031 & $=0.6 \times \mathrm{MHD}-0.5$ & 0.76 & $<0.001$ \\
\hline LV lateral wall & $=1.0 \times \mathrm{MHD}-0.9$ & 0.81 & $<0.001$ & $=0.6 \times \mathrm{MHD}-0.7$ & 0.94 & $<0.001$ \\
\hline LV inferior wall & $=0.6 \times M H D-0.3$ & 0.76 & $<0.001$ & $=0.6 \times M H D-0.7$ & 0.87 & $<0.001$ \\
\hline LV septal wall & $=0.9 \times M H D-0.1$ & 0.85 & $<0.001$ & $=0.9 \times M H D-0.9$ & 0.95 & $<0.001$ \\
\hline Right ventricle & $=1.0 \times M H D+0.2$ & 0.80 & $<0.001$ & $=1.0 \times M H D-0.6$ & 0.92 & $<0.001$ \\
\hline Left auricle & $=0.6 \times \mathrm{MHD}+0.3$ & 0.70 & $<0.001$ & $=1.0 \times \mathrm{MHD}-0.7$ & 0.91 & $<0.001$ \\
\hline Right auricle & $=0.5 \times M H D+0.8$ & 0.77 & $<0.001$ & $=1.3 \times \mathrm{MHD}-0.3$ & 0.80 & $<0.001$ \\
\hline
\end{tabular}

b. Linear models for coronary artery segmentation

\begin{tabular}{|c|c|c|c|c|c|c|}
\hline & \multicolumn{6}{|c|}{ Coronary artery segmentation } \\
\hline & \multicolumn{3}{|c|}{ Left-sided breast irradiation } & \multicolumn{3}{|c|}{ Right-sided breast irradiation } \\
\hline & Mean dose & $r$ & p value & Mean dose & $\mathbf{r}$ & p value \\
\hline Left main coronary artery & $=0.8 \times \mathrm{MHD}+1.0$ & 0.59 & 0.001 & $=1.0 \times \mathrm{MHD}+0.3$ & 0.77 & $<0.001$ \\
\hline Left anterior descending artery (LADCA) & $=2.6 \times M H D-1.2$ & 0.64 & $<0.001$ & $=0.8 \times M H D+0.3$ & 0.73 & 0.001 \\
\hline LADCA proximal segment & $=2.4 \times \mathrm{MHD}-5.3$ & 0.70 & $<0.001$ & $=0.7 \times M H D+1.3$ & 0.70 & 0.001 \\
\hline LADCA mid segment & $=2.9 \times \mathrm{MHD}-1.3$ & 0.53 & 0.004 & $=0.9 \times \mathrm{MHD}+0.7$ & 0.55 & 0.007 \\
\hline LADCA distal segment & $=2.0 \times \mathrm{MHD}+5.5$ & 0.27 & 0.069 & $=0.8 \times M H D-0.6$ & 0.68 & 0.001 \\
\hline Circumflex artery (CxA) & $=0.8 \times \mathrm{MHD}-0.7$ & 0.86 & $<0.001$ & $=0.7 \times \mathrm{MHD}-0.5$ & 0.86 & $<0.001$ \\
\hline CXA proximal segment & $=1.0 \times \mathrm{MHD}-0.5$ & 0.65 & $<0.001$ & $=0.8 \times \mathrm{MHD}-0.2$ & 0.76 & $<0.001$ \\
\hline CXA distal segment & $=0.7 \times M H D-1.0$ & 0.88 & $<0.001$ & $=0.6 \times M H D-0.5$ & 0.83 & $<0.001$ \\
\hline Right coronary artery (RCA) & $=0.7 \times M H D+0.9$ & 0.52 & 0.004 & $=1.2 \times M H D-0.2$ & 0.86 & $<0.001$ \\
\hline RCA proximal segment & $=0.6 \times \mathrm{MHD}+3.3$ & 0.24 & 0.070 & $=1.6 \times \mathrm{MHD}+1.3$ & 0.76 & $<0.001$ \\
\hline RCA mid segment & $=0.5 \times \mathrm{MHD}+2.8$ & 0.09 & 0.215 & $=1.5 \times \mathrm{MHD}-1.5$ & 0.81 & $<0.001$ \\
\hline RCA distal segment & $=0.5 \times M H D+0.2$ & 0.50 & 0.004 & $=0.9 \times \mathrm{MHD}-1.0$ & 0.72 & 0.001 \\
\hline RCA posterior descending segment & $=1.1 \times M H D-1.9$ & 0.67 & $<0.001$ & $=0.6 \times \mathrm{MHD}-0.6$ & 0.78 & $<0.001$ \\
\hline
\end{tabular}

$\mathrm{r}$ is the correlation coefficient, $\mathrm{p}$ values are adjusted for multiple testing with Holm-Bonferroni method. 
routinely delineated. This approach, illustrated herein for breast rotational IMRT, can be extrapolated to other clinical situations where cardiac radiation exposure is concerned.

And yet, these linear models based on MHD must be used with some caution. While correlations between MHD and mean dose to cardiac substructures were strong for right-sided irradiations, they were more moderate for left-sided irradiations: in this case, a part of the substructure exposure cannot be explained by MHD variation, generating an uncertainty in its estimation. In addition, MHD provides no information on high doses to cardiac substructures. The histological diversity of the cardiac substructure (muscle, nerves, epithelial tissues, and lymphatic and blood tissue) explains the broad range of described radiation-induced adverse cardiac events [4]: specific cardiac substructure doses may thus be more important than MHD. For instance, atrial dose correlates with specific cardiotoxicity for lung cancer patients [5-6]. Even though substructure dose constraints are not precisely known yet, toxicity studies taking into account cardiac segmentation may help clarifying them.

\section{Conclusions}

Clinical practice and cardiotoxicity trials would gain by considering cardiac substructure exposure; relying solely on the MHD for cardiac sparing may be simplistic, but linear regression models can help in estimating cardiac substructure exposure when only MHD is available.
Conflict of interest: none declared

\author{
Pierre Loap \\ Institut Curie \\ 26 rue d'ulm \\ 75006 Paris, France \\ e-mail:pierre.loap@gmail.com
}

Received: 7 Mar 2020

Accepted: 27 Mar 2020

\section{References}

1. Effect of radiotherapy after mastectomy and axillary surgery on 10-year recurrence and 20-year breast cancer mortality: meta-analysis of individual patient data for 8135 women in 22 randomised trials. The Lancet. 2014; 383(9935): 2127-2135, doi: 10.1016/s0140-6736(14)60488-8.

2. Lauche O, Kirova YM, Fenoglietto $P$, et al. Helical tomotherapy and volumetric modulated arc therapy: New therapeutic arms in the breast cancer radiotherapy. World J Radiol. 2016; 8(8): 735-742, doi: 10.4329/ wjr.v8.i8.735, indexed in Pubmed: 27648167.

3. Duane F, Aznar MC, Bartlett F, et al. A cardiac contouring atlas for radiotherapy. Radiother Oncol. 2017; 122(3): 416-422, doi: 10.1016/j. radonc.2017.01.008, indexed in Pubmed: 28233564.

4. Piroth MD, Baumann R, Budach $W$, et al. Heart toxicity from breast cancer radiotherapy : Current findings, assessment, and prevention. Strahlenther Onkol. 2019; 195(1): 1-12, doi: 10.1007/s00066-018-1378z, indexed in Pubmed: 30310926.

5. Stam B, Peulen $\mathrm{H}$, Guckenberger M, et al. Dose to heart substructures is associated with non-cancer death after SBRT in stage I-II NSCLC patients. Radiother Oncol. 2017; 123(3): 370-375, doi: 10.1016/j. radonc.2017.04.017, indexed in Pubmed: 28476219.

6. Reshko LB, Kalman NS, Hugo GD, et al. Cardiac radiation dose distribution, cardiac events and mortality in early-stage lung cancer treated with stereotactic body radiation therapy (SBRT). J Thorac Dis. 2018; 10(4): 2346-2356, doi: 10.21037/jtd.2018.04.42, indexed in Pubmed: 29850140 . 